\title{
Benchmarking the new ENDF/B-VIII.0 nuclear data library for OECD/NEA medium 1000 MWth sodium-cooled fast reactor
}

\author{
Donny Hartanto ${ }^{1, *}$, Bassam Khuwaileh ${ }^{1}$, and Peng Hong Liem Ki, $^{2,3}$ \\ ${ }^{1}$ University of Sharjah, United Arab Emirates \\ ${ }^{2}$ Tokyo City University, Japan \\ ${ }^{3}$ Nippon Advanced Information Service (NAIS Co., Inc.), Japan
}

\begin{abstract}
This paper presents the benchmark evaluation of the new ENDF/B-VIII.0 nuclear library for the OECD/NEA Medium 1000 MWth Sodium-cooled Fast Reactor (SFR). There are 2 SFR cores: metallic fueled (MET-1000) and oxide fueled (MOX-1000). The continuous-energy Monte Carlo Serpent 2 code was used as the calculation tool. Various nuclear libraries such as ENDF/B-VII.1 and JENDL-4.0 were included to be compared with the newest ENDF/B-VIII.0. The evaluated parameters are $k, \beta_{e f f}$, sodium void reactivity $\left(\Delta \rho_{N a}\right)$, Doppler constant $\left(\Delta \rho_{\text {Doppler }}\right)$, and control rod worth $\left(\Delta \rho_{C R}\right)$.
\end{abstract}

\section{Introduction}

Under the working party of OECD/NEA (Organisation for Economic Co-operation and Development/Nuclear Energy Agency), the neutronics benchmark study of the Generation-IV sodium-cooled fast reactor (SFR) concepts has been performed and published using different methods and codes [1-3] in conjunction with various nuclear data libraries such as ENDF/B-VII.0 [4], ENDF/B-VII.1 [5], JEFF-3.1 [6], and JENDL-4.0 [7]. The benchmark consists of large $3600 \mathrm{MW}$ th carbide and oxide cores, and also medium $1000 \mathrm{MWth}$ metallic and oxide cores. In this paper, the medium SFR cores are calculated by using the new version of nuclear data library, ENDF/B-VIII.0 [8], which has been released in 2018. The newer library has improvement on many neutron cross section libraries including the cross sections of materials which are commonly used in the SFR such U-235 and Pu-239 in the fuel, Fe-56 in the clad, and O-16 for mixed oxide fuel.

Since accuracy of the nuclear data is very important for the design and safety of a nuclear reactor, the impact of the new ENDF/B-VIII.0 on the neutronics and kinetics parameters of the OECD/NEA Medium 1000 MWth SFR is investigated. Both metallic and oxide cores are considered to distinguish the impact of the neutron spectrum. Several important neutronics parameters are evaluated such as effective neutron multiplication factor $k$, total effective delayed neutron fraction $\beta_{\text {eff }}$, prompt neutron generation time $\Lambda$, Doppler konstant $K_{D}$, coolant void reactivity (CVR), and control rod (CR) worth. Each parameter is calculated at beginning of cycle (BOC) and end of cycle (EOC) using the continuous-energy Monte Carlo Serpent2 code [9]. Three different modern nuclear data libraries are considered which are ENDF/B-VIII.0, ENDF/B-VII.1,

*e-mail: dhartanto@sharjah.ac.ae and JENDL-4.0. Beside inter-library comparison, the calculated results are also compared to the result reported by the working group [3]. It should be noted that the reported result is an average result computed by different calculation methods and various nuclear data libraries.

\section{Core Description}

The layout of the metallic core is illustrated in Fig. 1. The core consists of inner and outer fuel regions, surrounded by reflector and shielding. Inner core region has 78 fuel sub-assemblies and outer core region has 102 fuel sub-assemblies. Meanwhile, there are 114 reflector sub-assemblies, 66 shielding sub-assemblies, 15 primary control sub-assemblies, and 4 secondary control subassemblies.

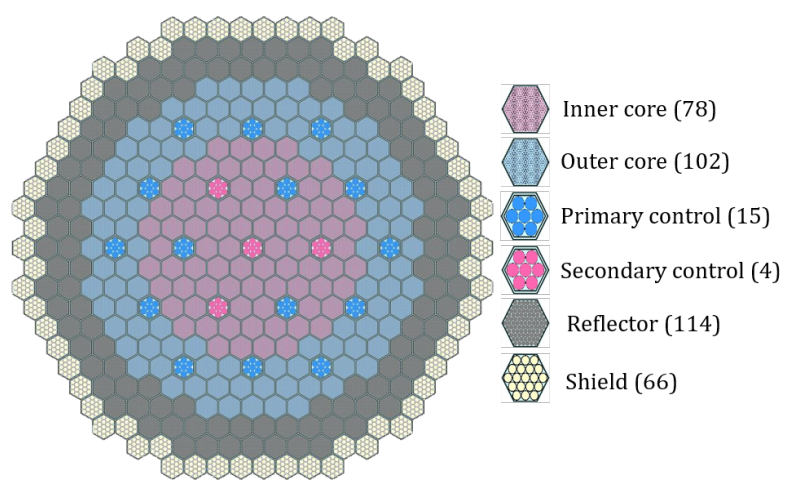

Figure 1. Radial Layout of Metallic Core [1]

The fuel consists of irradiated U-10Zr metallic fuel and it is contained in HT-9 cladding. The total core height is 480.20 , while the active core height is about $85.82 \mathrm{~cm}$. 
In the calculation, the temperature of the fuel is $534^{\circ} \mathrm{C}$, and the temperature of coolant and structural material is $432.5^{\circ} \mathrm{C}$

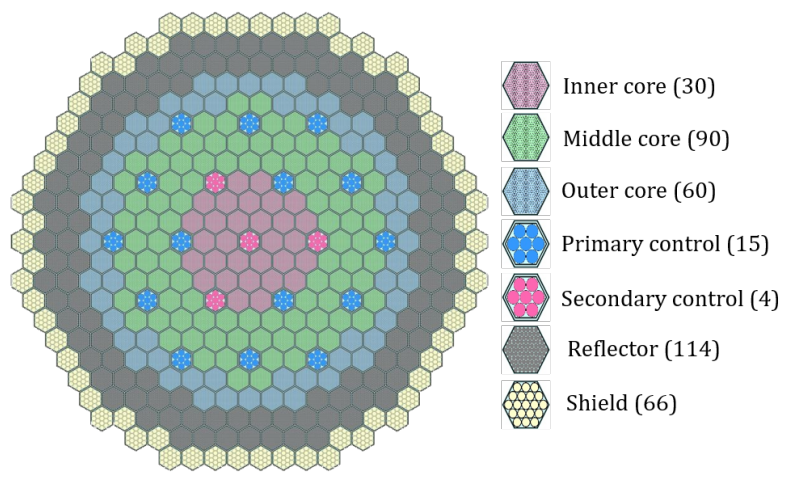

Figure 2. Radial Layout of Oxide Core [1]

In Fig. 2, the radial layout of the oxide core is shown. The core has 3 fuel regions which are inner, mid, and outer regions. The fuel is irradiated $\mathrm{UO}_{2}-(\mathrm{TRU})-\mathrm{O}_{2}$ contained in HT-9 clad. There are 30 inner fuel sub-assemblies, 90 mid fuel sub-assemblies, and 60 outer fuel sub-assemblies. Meanwhile the number of non-fuel sub-assemblies is same as in the metallic core. The total core height is also same as in the metallic core, however the active core is slightly taller, about $114.94 \mathrm{~cm}$. The fuel temperature is also higher, about $1027^{\circ} \mathrm{C}$, and the other components have same temperatures as in metallic core. For detail information on the benchmark including the material compositions and assembly dimension, one can refer to [1].

\section{Results and Discussion}

The Monte Carlo Serpent2 code was used to produce the subsequent results. In the calculation, the sub-assembly in the core was explicitly modeled. Each calculation used 100,000 neutron histories and 1,050 total neutron cycles with 50 inactive ones. Using this calculation condition, the standard deviation of $k$ is about $7 \mathrm{pcm}$.

The effective neutron multiplication factor $k$ values for metallic and oxide cores are summarized in Tables 1 and 2 , respectively. Comparing among the different libraries, JENDL-4.0 provides the highest $k$ value of the metallic core at BOC and EOC than the ones by ENDF/BVII.0 and ENDF/B-VII.1. Meanwhile, both JENDL-4.0 and ENDF/B-VII.1 produce similar $k$ and they are higher than the one by ENDF/VIII.0 for oxide core. It is noted that the calculated $k$ values are lower than the ones reported [3]. Additional analysis to evaluate the impact of ENDF/B-VIII.0 library was also conducted by replacing a single isotope from the old library by a new one from ENDF/B-VIII.0 library at BOC, summarized in Tables 3 and 4. It is noticed that there is significant $k$ changes from Fe-56 and U-238 of ENDF/B-VII.1 and Fe-56 and Pu-239 of JENDL-4.0 for both cores.

Figures 3 and 4 show the sensitivity coefficient of $k$ for metallic and oxide cores respectively. The sensitivity
Table 1. $k$ of metallic core at $\mathrm{BOC}$ and $\mathrm{EOC}$

\begin{tabular}{lcc}
\hline Library & BOC & EOC \\
\hline ENDF/B-8.0 & $1.02993 \pm 0.00007$ & $1.00580 \pm 0.00007$ \\
ENDF/B-7.1 & $1.02808 \pm 0.00007$ & $1.00522 \pm 0.00007$ \\
JENDL-4.0 & $1.03240 \pm 0.00007$ & $1.00893 \pm 0.00007$ \\
\hline Reported [3] & $1.03578 \pm 0.0078$ & $1.01280 \pm 0.0071$ \\
\hline
\end{tabular}

Table 2. $k$ of oxide core at BOC and EOC

\begin{tabular}{lcc}
\hline Library & BOC & EOC \\
\hline ENDF/B-8.0 & $1.02362 \pm 0.00006$ & $1.00567 \pm 0.00006$ \\
ENDF/B-7.1 & $1.02610 \pm 0.00006$ & $1.00857 \pm 0.00006$ \\
JENDL-4.0 & $1.02539 \pm 0.00006$ & $1.00876 \pm 0.00007$ \\
\hline Reported [3] & $1.02860 \pm 0.0062$ & $1.01360 \pm 0.0082$ \\
\hline
\end{tabular}

Table 3. Impact of the specific isotope library from ENDF/B-VIII.0 to $k$ of metallic core at BOC

\begin{tabular}{ccc}
\hline Old Library & $\begin{array}{c}\text { Library from } \\
\text { ENDF/B-8.0 }\end{array}$ & $\begin{array}{c}\text { Change } \\
{[\mathrm{pcm}]}\end{array}$ \\
\hline ENDF/B-7.1 & Na-23 & $14 \pm 7$ \\
& Fe-56 & $\mathbf{- 4 6 6} \pm \mathbf{7}$ \\
& U-235 & $6 \pm 7$ \\
& U-238 & $\mathbf{2 6 6} \pm \mathbf{7}$ \\
& Pu-239 & $86 \pm 7$ \\
& Am-241 & $8 \pm 7$ \\
\hline JENDL-4.0 & Na-23 & $68 \pm 7$ \\
& Fe-56 & $\mathbf{- 2 8 1} \pm \mathbf{7}$ \\
& U-235 & $15 \pm 7$ \\
& U-238 & $32 \pm 7$ \\
& Pu-239 & $\mathbf{- 1 4 5} \pm \mathbf{7}$ \\
& Am-241 & $-94 \pm 7$ \\
\hline
\end{tabular}

Table 4. Impact of the specific isotope library from ENDF/B-VIII.0 to $k$ of oxide core at BOC

\begin{tabular}{ccc}
\hline Old Library & $\begin{array}{c}\text { Library from } \\
\text { ENDF/B-8.0 }\end{array}$ & $\begin{array}{c}\text { Change } \\
{[\mathrm{pcm}]}\end{array}$ \\
\hline ENDF/B-7.1 & O-16 & $-33 \pm 9$ \\
& Na-23 & $-8 \pm 9$ \\
& Fe-56 & $\mathbf{- 5 6 9} \pm \mathbf{9}$ \\
& U-235 & $-14 \pm 9$ \\
& U-238 & $\mathbf{2 0 5} \pm \mathbf{9}$ \\
& Pu-239 & $-42 \pm 9$ \\
& Am-241 & $-10 \pm 9$ \\
\hline JENDL-4.0 & O-16 & $16 \pm 9$ \\
& Na-23 & $96 \pm 9$ \\
& Fe-56 & $\mathbf{- 3 4 8} \pm \mathbf{9}$ \\
& U-235 & $13 \pm 9$ \\
& U-238 & $78 \pm 9$ \\
& Pu-239 & $\mathbf{- 2 8 8} \pm \mathbf{9}$ \\
& Am-241 & $-65 \pm 9$ \\
\hline
\end{tabular}


coefficients are consistent among different nuclear data libraries. It is noticed that Pu-239 (n,f), U-238 (n,f), Pu-240 $(\mathrm{n}, \mathrm{f})$, and $\mathrm{Pu}-241$ (n,f) give the large positive sensitivities for both cores. Meanwhile, U-238 (n,g) and (n,inl) dominate the negative sensitivities for metallic core. On the other hand, the large negative sensitivities for oxide core include U-238 (n,g) and (n,inl), Pu-239 (n,g), O-16 (n,el), Pu-240 (n,g), and Fe-56 (n,inl).

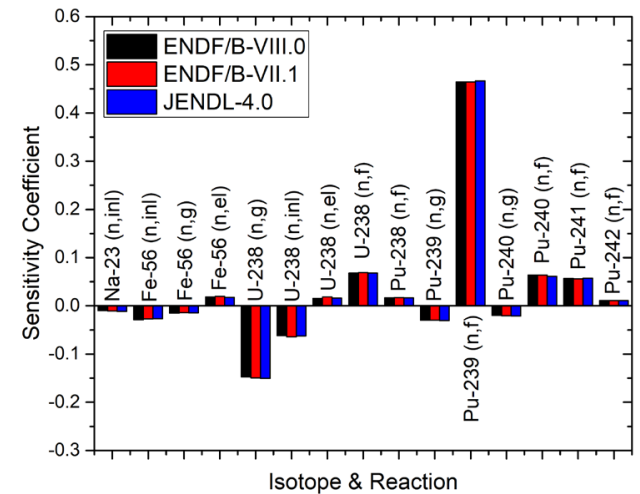

Figure 3. Sensitivity coefficient of metallic core for different nuclear data libraries

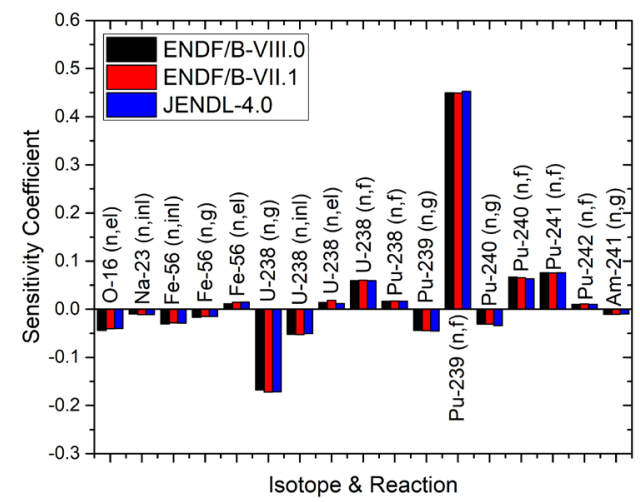

Figure 4. Sensitivity coefficient of oxide core for different nuclear data libraries

The calculated kinetic parameters $\Lambda$ and $\beta_{\text {eff }}$ at BOC and EOC are shown in Figs. 5 and 6 respectively. The parameters are adjoint-weighted value, calculated using the infinite fission probability method implemented in Serpent2. The $\Lambda$ of metallic core is shorter than the one of oxide core due to the harder spectrum in the metallic core, while the $\beta_{\text {eff }}$ of the metallic core is higher than the one of oxide core due to less amount of $\mathrm{Pu}$ and minor actinide in the metallic core. It is noted that the three different libraries produce similar and consistent kinetic parameters. Meanwhile, the reported $\beta_{\text {eff }}$ [3] is slightly higher than the calculated values.

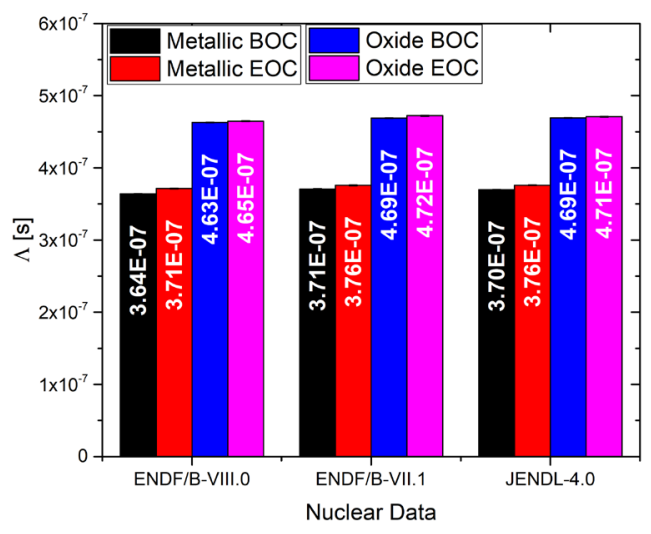

Figure 5. $\Lambda$ of both cores for different nuclear data libraries

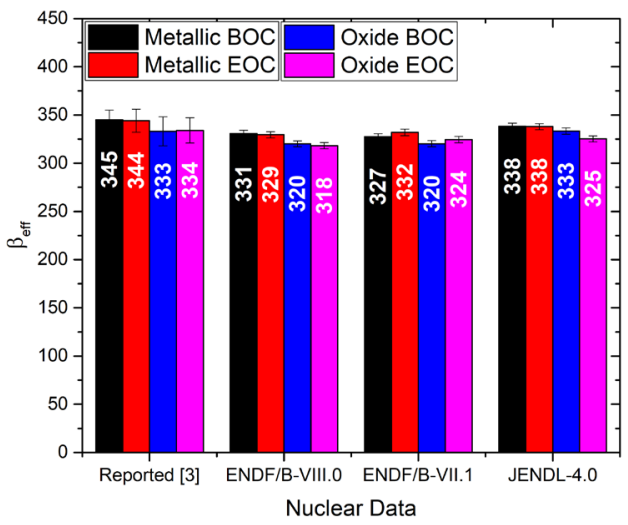

Figure 6. $\beta_{\text {eff }}$ of both cores for different nuclear data libraries

Figure 7 shows the Doppler constant $K_{D}$ for both cores at BOC and EOC. It is defined as the reactivity difference when the fuel temperature is doubled and at normal fuel operating temperature. It is clearly shown that the oxide core has more negative $K_{D}$ than the metallic core due to the softer spectrum in the oxide core. Moreover, the oxide core contains more U-238 than in metallic core. It is also noted that the similar and consistent $K_{D}$ values are given by the three different libraries. Meanwhile, the reported $K_{D}$ [3] is also slightly higher than the calculated ones.

The coolant void reactivity (CVR) is summarized in Figure 8 for both cores at BOC and EOC. It is defined as the reactivity difference when the coolant is voided and at normal condition. It is clearly shown that the metallic core has more positive (CVR) than the oxide core due to the harder spectrum in the metallic core. It is also noted that the similar and consistent CVR values are given by the three different libraries. Meanwhile, the reported CVR values [3] is lower than the calculated ones except for the oxide core by ENDF/B-VII.1.

The last result which is the control rod (CR) worth is shown in Fig. 9. It is calculated as the reactivity differ- 


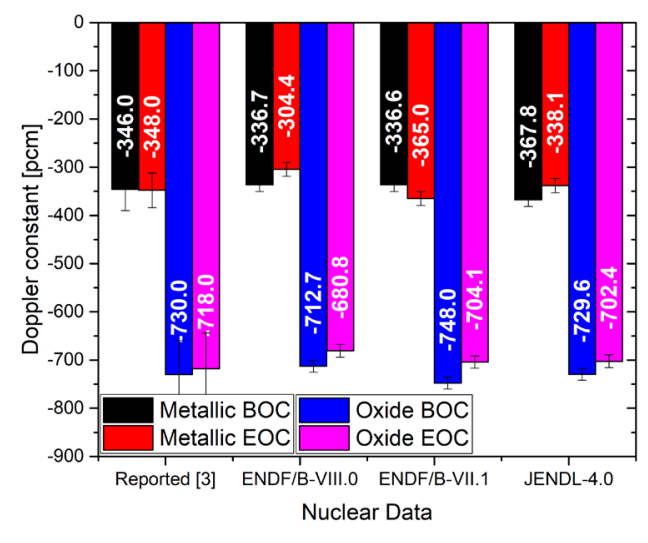

Figure 7. $K_{D}$ of both cores for different nuclear data libraries

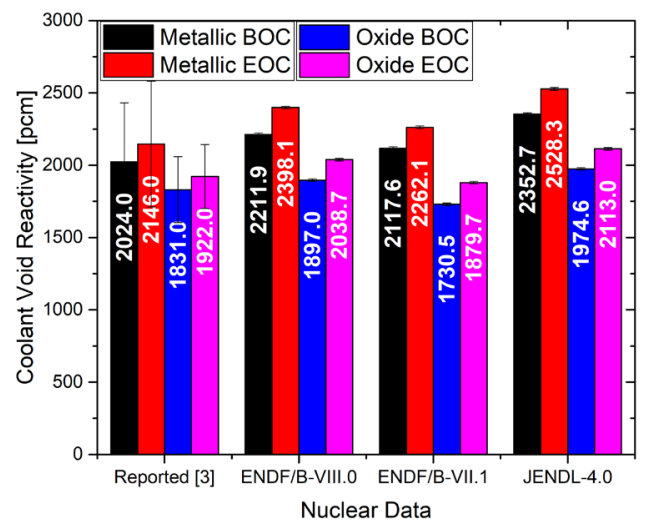

Figure 8. CVR of both cores for different nuclear data libraries

ence when all of the control sub-assemblies are withdrawn and inserted. It is also noted that the similar and consistent CR worth values are given by the three different libraries. Meanwhile, the reported CR worth values [3] is higher than the calculated ones.

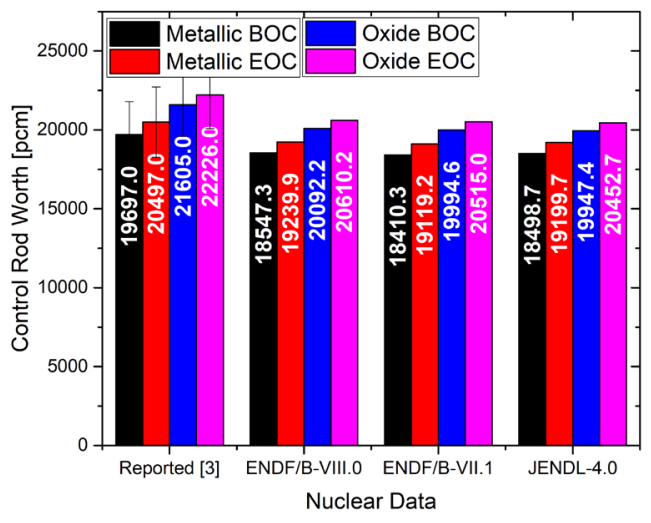

Figure 9. CR worth of both cores for different nuclear data libraries

\section{Conclusion}

Impact of the new released ENDF/B-VIII.0 nuclear data library to the neutronics and kinetics parameters of 2 different spectra SFR cores (metallic and oxide) has been studied. The new released Fe-56 cross section especially its (n, inl) and (n, g) cross sections shows significant reduction on $k$ compared to the ones with ENDF/B-VII.1 and JENDL-4.0. Another significant impact includes the U-238 of ENDF/B-VII.1 and Pu-239 of JENDL-4.0. On the other hand, the values of kinetics parameters, Doppler constant, coolant void reactivity, and control rod worth between libraries are consistent.

\section{References}

[1] NEA, Benchmark for Neutronic Analysis of Sodiumcooled Fast Reactor Cores with Various Fuel Types and Core Sizes, NEA/NSC/R(2015)9, OECD (2016)

[2] L. Buiron et al., Evaluation of Large 3600MWth Sodium-cooled Fast Reactor OECD Neutronic Benchmarks, Proc. Int. Conf. PHYSOR2014, Kyoto, Japan (2014)

[3] N.E. Stauff et al., Evaluation of Medium 1000MWth Sodium-cooled Fast Reactor OECD Neutronic Benchmarks, Proc. Int. Conf. PHYSOR2014, Kyoto, Japan (2014)

[4] M.B. Chadwick et al., Nucl. Data Sheets 107 (12), 2931-3060 (2006)

[5] M.B. Chadwick et al., Nucl. Data Sheets 112 (12), 2887-2996 (2011)

[6] A. Koning et al. (Ed.), The JEFF-3.1 Nuclear Data Library, NEA No. 6190, OECD (2006).

[7] K. Shibata et al., J. Nucl. Sci. Technol. 48 (1), 1-30 (2011)

[8] D.A. Brown et al., Nucl. Data Sheets 148, 1-142 (2018)

[9] J. Leppänen et al, Ann. Nucl. Energy 82, 142-150 (2015) 